\title{
Discriminant analysis for predictor of falls in stroke patients by using the Berg Balance Scale
}

\author{
Noriaki Maeda ${ }^{1}$, PT, PhD, Yukio Urabe ${ }^{1}$, PT, PhD, Masahito Murakami ${ }^{2}$, PT, PhD, Keisuke $\underline{\text { Itotani }}^{2}$, PT, Junichi $\underline{\text { Kato }}^{3}$, MD, PhD
}

\begin{abstract}
INTRODUCTION An observational study was carried out to estimate the strength of the relationships among balance, mobility and falls in hemiplegic stroke inpatients. The objective was to examine factors that may aid in the prediction of the likelihood of falls in stroke patients.

METHODS A total of 53 stroke patients (30 male, 23 female) aged $67.0 \pm 11.1$ years were interviewed regarding their fall history. Physical performance was assessed using the Berg Balance Scale (BBS) and the Functional Independence Measure (FIM) scale. Variables that differed between fallers and non-fallers were identified, and a discriminant function analysis was carried out to determine the combination of variables that effectively predicted fall status.

RESULTS Of the 53 stroke patients, 19 were fallers. Compared with the non-fallers, the fallers scored low on the FIM, and differed with respect to age, time from stroke onset, length of hospital stay, Brunnstrom recovery stage and admission BBS score. Discriminant analysis for predicting falls in stroke patients showed that admission BBS score was significantly related to the likelihood of falls. Moreover, discriminant analysis showed that the use of a significant BBS score to classify fallers and non-fallers had an accuracy of $81.1 \%$. The discriminating criterion between the two groups was a score of 31 points on the BBS.

CONCLUSION The results of this study suggest that BBS score is a strong predictor of falls in stroke patients. As balance is closely related to the risk of falls in hospitalised stroke patients, BBS might be useful in the prediction of falls.
\end{abstract}

Keywords: Berg Balance Scale, discriminant, falls, Functional Independence Measure, stroke

\section{INTRODUCTION}

Falls are relatively common among hospitalised inpatients and are a major problem in stroke rehabilitation. ${ }^{(1,2)}$ An incidence of falls between $23 \%$ and $50 \%$ has been reported in studies on stroke patients. ${ }^{(3)}$ Prevention of falls should be a goal of acute, rehabilitative and chronic stroke care.

Previous studies have shown that impaired balance, ${ }^{(4,5)}$ functional impairment and cognitive deficit are related to the incidence of falls in patients with acute and subacute stroke. ${ }^{(6-9)}$ Hyndman et al reported that disturbances in balance increase the risk of falls. ${ }^{(10)}$ Teasell et al reported that significant differences were observed between non-fallers and fallers in terms of the presence of apraxia and defective cognitive function; they also reported that stroke patients with reduced cognitive function had difficulty following instructions to ensure safety in walking and transfer. ${ }^{(11)}$ Not all studies concur with these findings; a study reported that balance ability is a relatively insignificant risk factor for falls. ${ }^{(6)}$

It is important to devise ways to prevent falls and to raise the performance status of stroke patients so as to prevent falls. However, it is also important to predict the occurrence of falls in order to prevent excessive limitation of activity. Methods to predict the occurrence of falls are based on individual experience, subjective observation and psychological assessment; only a few studies have established a standardised criterion for the prediction of falls. ${ }^{(6,10)}$
The objective of the present study was to examine the factors that contribute to falls in stroke inpatients who have chronic disabilities. This was done by investigating the fallers' and non-fallers' physical characteristics, Functional Independence Measure (FIM) scores on admission and discharge, ${ }^{(12)}$ Berg Balance Scale (BBS) scores on admission ${ }^{(13)}$ and mini-mental state examination (MMSE) scores. ${ }^{(14)}$ The variables that were best able to discriminate between the two groups were determined.

\section{METHODS}

The present study was approved by the Internal Clinical Ethics Committee of Hyogo Prefectural Rehabilitation Center at Nishiharima, Japan. A total of 53 stroke patients (30 male, 23 female; mean age $67.0 \pm 11.1$ years) who were admitted to Hyogo Prefectural Rehabilitation Center at Nishi-harima, Japan, were included in the study. The nature of the study was explained to the patients and their informed consent was obtained. Among the 53 patients, 32 were diagnosed with intracerebral infarction, 15 were diagnosed with intracerebral haemorrhage and 6 were diagnosed with subarachnoid haemorrhage. The nature of the paralysis was right hemiplegia in 28 patients, left hemiplegia in 23 patients and quadriplegia in 2 patients. The mean duration of the paralysis was $27.2 \pm 31.3$ months after stroke onset. The mean length of hospital stay (LOS) was $79.4 \pm 39.6$ days. The exclusion criteria were: (a) a history of major musculoskeletal problems (e.g. amputation or recent joint replacement surgery);

${ }^{1}$ Department of Sport Rehabilitation, Graduate of Biomedical \& Health Sciences, Hiroshima University, Hiroshima, ${ }^{2}$ Department of Physical Therapy, Faculty of Rehabilitation, Kobe International University, Kobe, Hyogo, ${ }^{3}$ Department of Internal Medicine, Hyogo Prefectural Rehabilitation Center at Nishi-harima, Tatsuno, Japan

Correspondence: Dr Noriaki Maeda, Assistant Professor, Department of Sport Rehabilitation, Graduate of Biomedical \& Health Sciences, Hiroshima University, Kasumi 1-2-3, Minami-ku, Hiroshima 734-8553, Japan. norimmi@hiroshima-u.ac.jp 
(b) transient ischaemic attacks and neurological disorders; (c) major psychiatric problems; and/or (d) intracranial operations. Following the definition by Gibson, ${ }^{(15)}$ the present study defined 'falls' as incidents during which a body region other than the soles involuntarily touches the floor or ground. This definition was used to categorise patients into fallers and non-fallers groups. All falls that occurred between the time of admission to and the time of discharge from the hospital were reported by medical staff using a dedicated fall report. These reports were the basis on which the patients were categorised into either the fallers (including both occasional fallers and repeat fallers) or non-fallers group.

The hospital records of the stroke patients were retrospectively reviewed to determine their age, gender, stroke type (i.e. ischaemic, haemorrhagic or subarachnoid haemorrhagic), time from stroke onset to admission, LOS, affected side of the body (i.e. right, left or bilateral), Brunnstrom recovery stage of the lower extremity on admission, FIM score on admission (i.e. total FIM, motor FIM and cognitive FIM), BBS score on admission and MMSE score on admission. The measurements of Brunnstrom recovery stage, and FIM, BBS and MMSE scores on admission were taken within five days from the date of admission for all patients. The relationship between patient characteristics and falls was investigated.

The Brunnstrom recovery stage of leg motor function was rated from III to $V$ in each patient. Brunnstrom described six temporal, stepwise stages that serve as a method for assessing motor function recovery in stroke patients with hemiplegia. ${ }^{(16)}$ The FIM instrument consists of 18 items (maximum of 126 points) under two major categories (i.e. motor and cognition); each of the 18 items are scored on an ordinal scale from 1 to 7 . The score is based on the level of assistance the patient needs in order to perform activities of daily living. A score of 1 indicates that the patient requires full assistance, while a score of 7 indicates total independence. A score of 0 is given if the activity does not occur at the time of admission (e.g. if it is unsafe for the patient to do the task). This tool has been shown to have good reliability and prognostic potential at admission. ${ }^{(17)}$

The BBS is a scale of functional balance comprising 14 items, each rated from 0 (i.e. poor balance) to 4 (i.e. good balance). The perfect score is 56 . To further standardise the test, the position for the 'forward reach' item in the BBS was altered slightly - the patients were required to raise both arms to an angle of $90^{\circ}$ and to keep their heels on the floor during the reach. ${ }^{(18)}$ The MMSE is a tool that assesses cognitive function and memorising ability. It is a cognitive screening test that comprises 11 items, which include orientation to place and time, registration, attention and concentration, recall, language (i.e. object naming, repetition, comprehension, reading, writing and three-step command) and visual construction (i.e. copying design). The maximum score is 30 points and the test takes about 5-10 minutes to administer.

The characteristics of the fallers and non-fallers were compared using Student's $t$-test to match parametric variables (i.e. age, time from stroke onset to admission, LOS, FIM score, BBS score and MMSE score). Chi-square test was used to compare gender, stroke type and affected side of the body, while Mann-Whitney $U$ test was applied to compare the Brunnstrom recovery stage for the lower extremities between the two groups. Pearson's correlation coefficient was used to determine the correlation among the variables (i.e. age, LOS, FIM score, BBS score and MMSE score).

Multiple regression analysis using forward stepwise regression was performed to evaluate the fall risk prediction, with BBS score as the dependent variable. To investigate the factors that independently affect the occurrence of falls, logistic regression analysis was performed, with the factors found to be significant on univariate analysis as the independent variables and the presence or absence of falls as the dependent variable. The receiver-operating-characteristic curve of the factors selected by logistic regression analysis was used to determine a cut-off value for accurate classification of fallers and non-fallers; the area under the curve was also calculated. All statistical analyses were performed using the Statistical Package for the Social Sciences for Windows (SPSS Inc, Chicago, IL, USA). A p-value $<0.05$ was considered statistically significant.

\section{RESULTS}

Of the 53 stroke patients, 19 (35.8\%) were fallers and 34 (64.2\%) were non-fallers. The characteristics of the fallers and non-fallers are compared in Table I. Comparison of the two groups showed that age $(p<0.001$, effect size $=0.49)$ and LOS $(p=0.003$, effect size $=0.28)$ were significantly higher in the fallers group; and Brunnstrom recovery stage on admission $(p=0.002$, effect size $=0.42)$, total FIM score on admission $(p<0.001$, effect size $=0.63)$, motor FIM score on admission ( $p<0.001$, effect size $=0.64)$, cognitive FIM score on admission $(p=0.006$, effect size $=0.38)$, BBS score on admission $(p<0.001$, effect size $=0.63)$, and MMSE score $(p=0.019$, effect size $=0.32$ ) were significantly lower in the fallers group. No significant differences were noted between the two groups in terms of their gender, stroke type, time from stroke onset to admission and affected side of the body.

Correlations among the variables related to falls are shown in Table II. A strong correlation was noted between total and motor FIM scores on admission, and BBS score on admission. Age, LOS, cognitive FIM score on admission, BBS score on admission and MMSE score were subjected to logistic regression analysis, with the occurrence of falls as the dependent variable.

The results of the discriminant function analyses are presented in Table III. Discriminant analysis was conducted to determine which fall factors best discriminated between the two groups of stroke patients (fallers vs. non-fallers). Box's M covariance test indicated equality between the matrices of both groups $(p=0.329)$. BBS score on admission was found to be the variable that best discriminated between stroke patients with a low risk of falls and those with a high risk of falls. BBS score on admission was found to be able to classify stroke patients into the faller or non-faller groups with an accuracy of $81.1 \%$ following validation (Wilks' $\lambda=0.50, p<0.001$ ) (Fig. 1). The discriminating criterion between the two groups was a score of 31 points on BBS.

\section{DISCUSSION}

Various internal and external multilevel risk factors have been reported to be associated with falls in stroke patients. ${ }^{(19)}$ In particular, the internal factor of reduced balance is a risk factor 
Table I. Comparison of the characteristics of the non-fallers and fallers.

\begin{tabular}{|c|c|c|c|c|}
\hline Characteristic & Non-fallers $(n=34)$ & Fallers $(n=19)$ & p-value & Effect size \\
\hline Age* $(y r)^{*}$ & $63.0 \pm 10.1$ & $74.2 \pm 9.2$ & $<0.001$ & 0.49 \\
\hline Gender ${ }^{+}$ & & & 0.097 & 0.08 \\
\hline Male & 22 & 8 & & \\
\hline Female & 12 & 11 & & \\
\hline Stroke type ${ }^{+}$ & & & 0.947 & 0.04 \\
\hline Ischaemic & 20 & 12 & & \\
\hline Haemorrhagic & 10 & 5 & & \\
\hline Subarachnoid haemorrhagic & 4 & 2 & & \\
\hline Time from stroke onset to admission* (mth) & $29.1 \pm 35.3$ & $23.8 \pm 23.2$ & 0.558 & 0.08 \\
\hline Length of hospital stay* (day) & $71.2 \pm 36.7$ & $94.1 \pm 41.3$ & 0.003 & 0.28 \\
\hline Affected side of the body ${ }^{+}$ & & & 0.101 & 0.16 \\
\hline Right & 17 & 11 & & \\
\hline Left & 15 & 8 & & \\
\hline Bilateral & 2 & 0 & & \\
\hline Brunnstrom recovery stage for lower extremity on admission* & V (IV-VI) & IV (III-V) & 0.002 & 0.42 \\
\hline \multicolumn{5}{|l|}{ Functional Independence Measure score on admission* } \\
\hline Total & $92.6 \pm 22.5$ & $55.9 \pm 21.2$ & $<0.001$ & 0.63 \\
\hline Motor & $66.8 \pm 18.2$ & $37.0 \pm 15.5$ & $<0.001$ & 0.64 \\
\hline Cognitive & $25.8 \pm 8.4$ & $18.9 \pm 8.3$ & 0.006 & 0.38 \\
\hline Berg Balance Scale score on admission* & $44.8 \pm 11.3$ & $19.8 \pm 20.1$ & $<0.001$ & 0.63 \\
\hline Mini-mental state examination score* & $21.4 \pm 8.2$ & $15.6 \pm 8.6$ & 0.019 & 0.32 \\
\hline
\end{tabular}

* Data presented as mean \pm standard deviation and Student's $t$-test was used to check for significance. ${ }^{\text {DData }}$ presented as number and chi-square test was used to check for significance. $¥$ Data presented as median (interquartile range) and Mann-Whitney $U$ test was used to check for significance.

Table II. Pearson's correlation coefficients for the variables evaluated for fall risk in hemiplegic stroke patients.

\begin{tabular}{|c|c|c|c|c|c|c|c|}
\hline Variable & Age & LOS & Total FIM* & Motor FIM* & Cognitive FIM* & BBS* $^{*}$ & MMSE \\
\hline Age & 1.000 & 0.100 & $-0.431^{+}$ & $-0.429^{+}$ & $-0.284^{*}$ & $-0.477^{+}$ & -2.224 \\
\hline LOS & - & 1.000 & $-0.431^{+}$ & $-0.439^{+}$ & $-0.258^{+}$ & $-0.418^{+}$ & -0.150 \\
\hline Total FIM* & - & - & 1.000 & $0.963^{+}$ & $0.739^{+}$ & $0.639^{+}$ & $0.462^{+}$ \\
\hline Motor FIM* & - & - & - & 1.000 & $0.531^{+}$ & $0.723^{+}$ & $0.308^{*}$ \\
\hline Cognitive FIM* & - & - & - & - & 1.000 & 0.200 & $0.687^{+}$ \\
\hline MMSE & - & - & - & - & - & - & 1.000 \\
\hline
\end{tabular}

Note: Some variables were highly correlated, and as such, they could not be entered simultaneously in the logistic regression analysis. * Score on admission. $\dagger p<0.01$ is considered statistically significant. $\neq p<0.05$ is considered statistically significant. BBS: Berg Balance Scale; FIM: Functional Independence Measure; MMSE: mini-mental stage examination

Table III. Results of the discriminant function analyses.

\begin{tabular}{lc}
\hline Variable & $\begin{array}{c}\text { Normalised } \\
\text { discriminant function }\end{array}$ \\
\hline BBS score on admission & $0.797^{*}$ \\
Age (yr) & -0.308 \\
Mini-mental state examination score & 0.289 \\
Cognitive FIM score on admission & 0.220 \\
Brunnstrom recovery on admission & -0.009 \\
Length of hospital stay (day) & 0.008 \\
\hline
\end{tabular}

* Step length parameter with higher discriminant value between groups. BBS: Berg Balance Scale; FIM: Functional Independence Measure

that has been found to be closely associated with falls. ${ }^{(20)}$ The aim of the present study was to collect data on stroke inpatients with chronic disabilities, so as to identify factors that predict the risk of falls and to establish a practical index for clinical practice.
Our comparison of the faller and non-faller groups revealed significant differences in their age, LOS, Brunnstrom recovery stage, FIM score on admission, BBS score on admission and MMSE score. Falls in stroke patients with chronic disabilities have been associated with poor balance and mobility. ${ }^{(10,19)}$ Several studies have shown that balance, ${ }^{(9,11)}$ activities of daily living ${ }^{(8,19)}$ and cognitive deficits are risk factors for falls in stroke patients. ${ }^{(9,19)}$ Several predictors of falls have also been identified in individuals in the acute and subacute phases of stroke recovery. ${ }^{(3,11)}$ In the present study, the total FIM score on admission and the motor FIM score on admission were strongly and positively correlated with the BBS score on admission. Bohannon and Leary also reported a positive correlation between BBS score on admission and FIM score on admission. ${ }^{(19)}$ Teasell et al ${ }^{(11)}$ reported that BBS scores on admission and FIM scores on admission were significantly lower among patients 


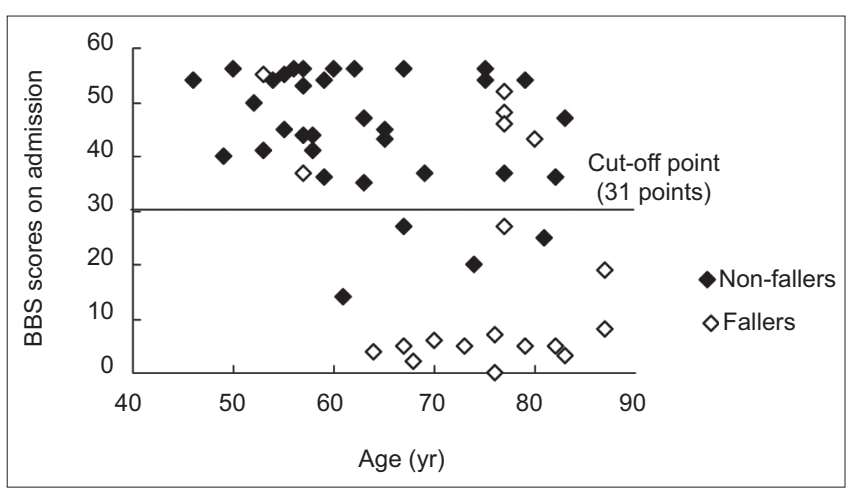

Fig. 1. Scatter chart shows the Berg Balance Scale (BBS) scores of the patients on admission. The horizontal line demonstrates the cut-off point.

who fell. A lower BBS score among fallers was expected, given that BBS is a measure of postural control in stroke patients and poor balance is associated with falls.

By performing a discriminant function analysis with the occurrence of falls as the dependent variable, we found that BBS score on admission was the variable that best discriminated between fallers and non-fallers. The findings suggest that BBS score on admission is an important factor for determining the risk of falls in stroke patients with chronic disabilities. In the present study, balance ability was determined using BBS, which describes the patient's degree of function. ${ }^{(21)}$ As a discriminant tool, BBS is also able to identify stroke patients who have difficulties in mobility associated with balance (i.e. patients who are at risk of future falls). ${ }^{(21)}$ In a previous study using BBS, Simpson et al reported that balance was the only common independent predictor of falls in individuals with and without a history of stroke. ${ }^{(22)}$ Their study indicated that the difference in the fall rates between the groups could be explained by the difference in their balance scores. ${ }^{(22)}$

In a previous study that used BBS, Kornetti et al proposed 45 points as the threshold value for predicting a high risk of falls associated with clinically impaired balance and transfer ability in the healthy elderly. ${ }^{(23)}$ In Shumway-Cook et al's report, the risk of falls in older adults was found to be high when the total BBS score was $\leq 36$; this risk increased by $6 \%-8 \%$ with every 1 -point reduction within the range of $46-54$ points. ${ }^{(24)}$ Simpson et al reported an association between the number of falls and BBS score in stroke patients who were discharged from a rehabilitation facility to their own homes in the community; BBS score was found to be useful for the prediction of falls - the number of falls increased sharply when the BBS score fell below 44. ${ }^{(22)}$ In the present study, the BBS score on admission was statistically lower than the cut-off value (i.e. 31 points). These findings were determined at the convalescence stage of the stroke patients, suggesting that reduced balance, low mobility and cognition are related to falls.

An advantage of the present study was that the evaluation of the BBS scores, together with the examination of the cut-off point on admission, reflected existing fears and concerns regarding falls in stroke patients. We found that the balance of stroke patients who are in convalescence to maintenance stages on admission was closely related to fall risk. As future intervention studies on fall prevention in stroke patients require the prediction of falls using objective evaluation indices, the accumulation of data on the changes in the physical characteristics of stroke patients may lead to the establishment of a reliable assessment method.

\section{REFERENCES}

1. Forster A, Young J. Incidence and consequences of falls due to stroke: a systematic inquiry. BMJ 1995; 311:83-6.

2. Mayo NE, Korner-Bitensky N, Becker R, Georges P. Predicting falls among patients in a rehabilitation hospital. Am J Phys Med Rehabil 1989; 68:139-46.

3. Stapleton T, Ashburn A, Stack E. A pilot study of attention deficits, balance control and falls in the subacute stage following stroke. Clin Rehabil 2001; 15:437-44.

4. Andersson AG, Kamwendo K, Seiger A, Appelros P. How to identify potential fallers in a stroke unit: validity indexes of 4 test methods. J Rehabil Med 2006; 38:186-91

5. Stolze $\mathrm{H}$, Klebe $\mathrm{S}$, Zechlin $\mathrm{C}$, et al. Falls in frequent neurological diseases-prevalence, risk factors and aetiology. J Neurol 2004; 251:79-84.

6. Jørgensen HS, Nakayama H, Raaschou HO, Olsen TS. Stroke. Neurologic and functional recovery the Copenhagen Stroke Study. Phys Med Rehabil Clin N Am 1999; 10:887-906.

7. Liu-Ambrose T, Pang MY, Eng JJ. Executive function is independently associated with performances of balance and mobility in communitydwelling order adults after mild stroke: implications for falls prevention. Cerebrovasc Dis 2007; 23:203-10.

8. Andersson AG, Kamwendo K, Appelros P. Fear falling in stroke patients: relationship with previous falls and functional characteristics. Int J Rehabil Res 2008; 31:261-4.

9. Jørgensen L, Engstad T, Jacobsen BK. Higher incidence of falls in long-term stroke survivors than in population controls: depressive symptoms predict falls after stroke. Stroke 2002; 33:542-7.

10. Hyndman D, Ashburn A. People with stroke living in the community: Attention deficits, balance, ADL ability and falls. Disabil Rehabil 2003; 25:817-22.

11. Teasell R, McRae M, Foley N, Bhardwaj A. The incidence and consequence of falls in stroke patients during inpatient rehabilitation: factors associated with high risk. Arch Phys Med Rehabil 2002; 83:329-33.

12. Granger CV, Hamilton BB. The Uniform Data System for Medical Rehabilitation report of first admission for 1991. Am J Phys Med Rehabil 1993; 72:33-8.

13. Berg KO. Measuring balance in the elderly: preliminary development of an instrument. Physiother Can 1989; 41:304-10.

14. Dick JP, Guiloff RJ, Stewart A, et al. Mini-mental state examination in neurological patients. J Neurol Neurosurg Psychiatry 1984; 47:496-9.

15. Gibson MJ. Falls in later life. In: Kane RL, Grimley-Evans J, MacFadyen D, eds. Improving the Health of Older People: A World View. New York: Oxford University Press, 1990: 296-315.

16. Sanford J, Moreland J, Swanson LR, Stratford PW, Gowland C. Reliability of the Fugl-Meyer assessment for testing motor performance in patients following stroke. Physical therapy 1993; 73:447-54.

17. Lamb SE, Ferrucci L, Volapto S, et al. Risk factors for falling in homedwelling older women with stroke: the Women's Health and Aging Study. Stroke 2003; 34:494-501.

18. Saverino A, Benevolo E, Ottonello M, Zsirai E, Sessarego P. Falls in a rehabilitation setting: functional independence and fall risk. Eura Medicophys 2006; 42:179-84.

19. Bohannon RW, Leary KM. Standing balance and function over the course of acute rehabilitation. Arch Phys Med Rehabil 1995; 76:994-6.

20. Kato-Narita EM, Nitrini R, Radanovic M. Assessment of balance in mild and moderate stages of Alzheimer's disease: implications on falls and functional capacity. Arq Neuropsiquiatr 2011; 69:202-7.

21. Goh EY, Chua SY, Hong SJ, Ng SS. Reliability and concurrent validity of Four Square Step Test scores in subjects with chronic stroke: a pilot study. Arch Phys Med Rehabil 2013; 94:1306-11.

22. Simpson LA, Miller WC, Eng JJ. Effect of stroke on fall rate, location and predictors: a prospective comparison of older adults with and without stroke. PLoS One 2011; 6:e19431.

23. Kornetti DL, Fritz SL, Chiu YP, Light KE, Velozo CA. Rating scale analysis of the Berg Balance Scale. Arch Phys Med Rehabil 2004; 85:1128-35.

24. Shumway-Cook A, Baldwin M, Polissar NL, Gruber W. Predicting the probability for falls in community-dwelling older adults. Phys Ther 1997; 77:812-9. 